\title{
Developing Application-Specific Multiprocessor Platforms on FPGAs
}

\author{
Sen Ma, Miaoqing Huang, David Andrews \\ Department of Computer Science and Computer Engineering \\ University of Arkansas \\ Fayetteville, Arkansas 72701, USA \\ \{senma,mqhuang,dandrews\}@uark.edu
}

\begin{abstract}
The capacity of FPGA devices has reached the 1-million-LUT level, which provides space to accommodate a complete Multiprocessor System-on-Chip on the programmable device. In this work we propose a design flow to create application-specific computation architecture based on the application entry in OpenCL. The application-specific system is based on a distributed memory hierarchy in which each processor is equipped with its own local memories for data and instructions. The multiple kernels in an application are mapped to individual processors to achieve the processing parallelism. In order to efficiently use the limited resource on FPGA devices, the sizes of local data and instruction memories are determined by analyzing the application source code. Compared with the traditional shared memory architectures, the application-specific platform is capable of reducing the resource requirement, providing more processing parallelism, and achieving higher performance.
\end{abstract}

Keywords-application-specific platform generation; FPGA; OpenCL.

\section{INTRODUCTION}

The capacity of FPGA devices has reached the 1-millionLUT milestone. For instance, a Xilinx Virtex-7V2000T device contains more than 1.2 million 6-input LUTs. This ample capacity makes it possible to put a complete MultiProcessor System-on-Programmable-Chip (MPSoPC) on a single FPGA device. A system consisting of 33 MicroBlaze processors has been implemented on a Xilinx Virtex6LX240T FPGA device [1]. As the performance of computing systems will come mainly from task level parallelism instead of instruction level parallelism, future MPSoPCs may contain 100's to 1000's processors in a single system.

Different from the way to develop applications on generalpurpose CPUs, the application development on the embedded system on FPGAs generally includes two steps, the hardware platform development and the design of the software running on the platform. Since these two steps are not closely integrated in the classic design flow, the hardware platform is not necessary the best fit for the corresponding software application. For example, the system design tools from the FPGA vendors are still generally based on the traditional shared memory architecture in which all the processors and system components are connected

This work was supported in part by Xilinx for equipment donation. to a single piece of global memory. Although the MultiPort Memory Controller (MPMC) on Xilinx FPGAs can be used to improve the access parallelism to the shared global memory, which is usually implemented on off-chip DRAMs, it only has 8 ports and implies quite a scalability limitation. Busses are well known to have the scalability issue, but they are still the primary communication subsystems used to integrate the components in a system. This separation between the platform design and the corresponding software development is not a great concern when there are just a few processors in the system. However, as the number of cores increases on FPGAs, the shared memory architecture and the bus will significantly limit the performance and scalability of systems consisting of tens to hundreds of processors. In order to get the best performance from the multiprocessor systems on FPGAs, it is desired to design applicationspecific hardware platforms considering the number of processors, the memory hierarchy, the interconnect, etc. In other words, the hardware platform needs to be designed to fit the needs of the application, which is generally written/modeled in high-level languages.

In this work, we present a design flow to develop application-specific platforms on large scale FPGAs. We use OpenCL [2] as the design entry. The platform is specific in the sense that the number of processors as well as the sizes of local memories are determined by profiling the corresponding application as follows. (1) Each OpenCL kernel will be deployed onto a separate processor to provide the maximum processing parallelism subject to the available resources on the FPGA device. (2) The application-specific hardware platform is based on the distributed memory hierarchy, which has demonstrated good scalability and performance [1]. Each processor has its own local instruction memory and data memory with appropriate size. In the design flow, an application written in OpenCL is first converted to a multithreaded C program, in which each OpenCL kernel is translated to a $\mathrm{C}$ function. In order to get the appropriate size of the local memories for each processor, the corresponding $\mathrm{C}$ function is compiled to machine code and the size of executable is measured to determine the volume of the local instruction memory. The size of the local data memory is determined by the explicit data transfer volume in the OpenCL code. By setting the sizes of the local memories properly, it becomes 


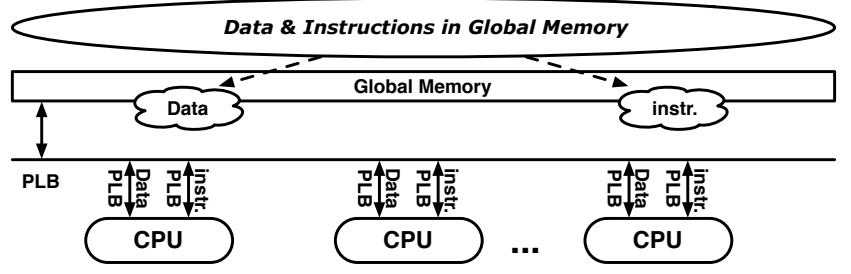

(a) Shared memory hierarchy.

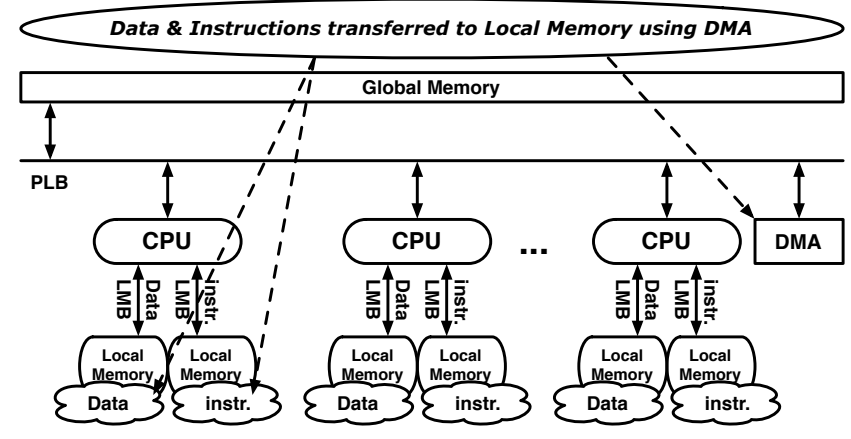

(b) Distributed memory hierarchy.

Figure 1. Comparison of two memory hierarchies (PLB: Processor Local Bus; LMB: Local Memory Bus).

possible to instantiate as many processors in the system as desired in addition to achieving a good performance.

The remainder of this paper is organized as follows. The basic knowledge of OpenCL, the distributed memory hierarchy, and the system infrastructure are briefly discussed in Section II. The design flow is presented in Section III. We use three benchmarks, matrix multiplication, 2D FFT, and Canny edge detection to demonstrate the benefit of the application-specific design flow compared with the application developed on traditional shared memory platforms. The results are shown in Section IV. Section V discusses the related work. The conclusions are given in Section VI.

\section{BACKGROUND}

The scalable performance of a multiprocessor system mainly comes from the coarse grained task parallelism among processors. OpenCL is a framework to design parallel applications on multicore/manycore platforms. Application development using OpenCL consists of two steps. The first step is to define a computing platform on which the application will execute. In OpenCL's term a platform consists of one host processor and multiple compute devices. The second step is to assign the computation tasks to compute devices and specify the dependencies among them through the explicit data transfer between these tasks. Each computation task is described in an OpenCL function called "kernel", which is assigned to one compute device during the runtime. The information of both the platform and the corresponding data affinity and parallelism are explicitly presented and easily extracted in OpenCL framework.

When an OpenCL application is implemented on an FPGA device, a computing system is generated by integrating multiple processors with other components in which the memory hierarchy plays a very important role for achieving the scalable performance. The traditional memory hierarchy of embedded systems is shown in Fig. 1(a) in which all the processors are connected to the same bus to access the shared memory. As the number of processors increases in the system, the main bus will become a bottleneck, greatly limiting the scalability of the system. The memory hierarchy used in the design flow to generate the applicationspecific platform is the distributed memory hierarchy shown in Fig. 1(b). Both instruction port and data port of each processor are connected to local memories through separate busses (e.g., Local Memory Bus on Xilinx FPGAs). In the distributed memory hierarchy, data and instructions are transferred from the global memory to the local memories using DMA engine before the data processing starts. The result data are transferred back to the global memory using DMA after the computation finishes. In this work the size of both memories is determined by analyzing the application in the platform generation process.

The computing platform is created based on the hthreads system [3]. The hthreads system consists of one host processor and a couple of worker processors, which naturally corresponds to the master-slave model of OpenCL applications. In hthreads system, part of the OS functionality, such as thread and mutex management, is handled by hardware microkernels to improve the efficiency and scalability.

\section{DESIGN FLOW}

As mentioned in Section I, the entry of the design flow is an application written in OpenCL. Our target device is Xilinx Virtex-6LX240T FPGA on which the MicroBlaze is used to implement the compute device, i.e., the worker processor. Therefore in this work the OpenCL kernel function is written in a sequential fashion targeting MicroBlaze scalar processor. The computing platform is generated based on hthreads system, on which the programming language is C. Given an application entry in OpenCL, the goal of the design flow is to generate the hardware platform and the corresponding executable running on the platform so that the performance can be maximized.

In order to maximize the processing parallelism of the system, each OpenCL kernel is scheduled to one worker processor subject to the available resource on the target 
Table I

THE REQUIRED SIZE OF THE LOCAL INSTRUCTION MEMORY AND THE LOCAL DATA MEMORY OF THREE BENCHMARKS.

\begin{tabular}{c||c|c||c||c|c||c|c|c}
\hline \multicolumn{3}{c||}{ Matrix Multiplication (MM) } & \multicolumn{3}{c||}{ 2D FFT* } & \multicolumn{3}{c}{ Canny Edge Detection*† } \\
\hline $\begin{array}{c}\text { Matrix } \\
\text { Size }\end{array}$ & $\begin{array}{c}\text { Instruction } \\
\text { Size (B) }\end{array}$ & $\begin{array}{c}\text { Data } \\
\text { Size (B) }\end{array}$ & $\begin{array}{c}\text { Matrix } \\
\text { Size }\end{array}$ & $\begin{array}{c}\text { Instruction } \\
\text { Size (B) }\end{array}$ & $\begin{array}{c}\text { Data } \\
\text { Size (B) }\end{array}$ & $\begin{array}{c}\text { Detection } \\
\text { Stage }\end{array}$ & $\begin{array}{c}\text { Instruction } \\
\text { Size (B) }\end{array}$ & $\begin{array}{c}\text { Data } \\
\text { Size (B) }\end{array}$ \\
\hline $2 \times 2$ & 1,964 & 48 & $2 \times 2$ & 2,316 & 16 & Stage 1 & 2,500 & 32,768 \\
\hline $4 \times 4$ & 2,004 & 192 & $4 \times 4$ & 2,784 & 64 & Stage 2 & 2,680 & 32,768 \\
\hline $8 \times 8$ & 2,004 & 768 & $8 \times 8$ & 2,884 & 256 & Stage 3 & 2,284 & 49,152 \\
\hline $16 \times 16$ & 2,004 & 3,072 & $16 \times 16$ & 3,072 & 1,024 & Stage 4 & 2,316 & 32,768 \\
\hline $32 \times 32$ & 2,004 & 12,288 & $32 \times 32$ & 3,248 & 4,096 & - & - & - \\
\hline $64 \times 64$ & 2,008 & 49,152 & $64 \times 64$ & 3,504 & 16,384 & - & - & - \\
\hline
\end{tabular}

${ }^{*}$ For matrix multiplication and 2D FFT, there are 6 worker processors. For Canny edge detection, there are 4 worker processors.

${ }^{\dagger}$ The image resolution is $128 \times 128$.

FPGA devices. Among the various resources on FPGA devices, BRAM modules are very precious since (1) they are used by many components such as MPMC and the MicroBlaze soft processor, and (2) there is a limited number of modules available on a single FPGA device. For example, on Xilinx Virtex-6LX240T, there are only 416 4-KB BRAM modules. In order to allocate as many processors as desired in a system, the size of each on-chip memory has to be carefully determined to use the least number of BRAM modules. In order to determine the proper size of the local instruction memory for each worker processor, the $\mathrm{xxd}$ tool in the Xilinx ISE suite is used to create a hex dump of the corresponding ELF file of a kernel. The necessary size of instruction memory is the size of the hex file. The size of the local data memory is obtained explicitly from the data transfer volume through the APIs between the host processor and the worker processors in OpenCL specification.

Because the OpenCL kernel function is written in a sequential fashion in this work, the translation from OpenCL to $\mathrm{C}$ is straightforward in the conversion from OpenCL kernel to $\mathrm{C}$ function. The data dependency among multiple $\mathrm{C}$ functions can be obtained from the explicit data forwarding between OpenCL kernels.

The design flow currently is semi-automated. We have developed a tool in Python that can convert the OpenCL application to $\mathrm{C}$ program running on the generated hthreads system [4]. The sizes of the local memories need to be manually collected at this moment. Once the size information is obtained, they are used to build the multiprocessor system with distributed memory hierarchy.

\section{Evaluation AND Results}

In this work we use three benchmarks to demonstrate the benefit of our design flow, i.e., (1) Matrix Multiplication (MM), (2) 2D fast Fourier transform, and (3) Canny edge detection [5]. In matrix multiplication, given two square matrices $\mathcal{A}$ and $\mathcal{B}, \mathcal{C}$ is calculated as $\mathcal{C}=\mathcal{A} \times \mathcal{B}$ in which each matrix entry is a 32-bit integer. In 2D FFT, given a square matrix $\mathcal{A}$, a $2 \mathrm{D}$ FFT is applied on it so that $\mathcal{B}=F(\mathcal{A})$ in which each matrix entry is a single-precision floating- point number. In the cases of matrix multiplication and 2D FFT, different worker processors deal with independent data sets so that they can run in parallel. For Canny edge detection, there are four stages in the edge detection process, i.e., Gaussian smoothing, Compass edge detecting, Non-maximum suppression, Edge tracing and thresholding. Therefore, 4 MicroBlaze processors are implemented to form a 4-stage pipeline, each processor working on one stage of the Canny edge detection algorithm.

The hardware platform is the Xilinx ML605 board including a Virtex-6LX240T FPGA device. MicroBlaze soft processors are used to implement both the host processor and the worker processors in the hthreads system. ISE suite 12.3 is used to build the MPSoPC on FPGA device.

We first carry out a straightforward comparison between the traditional platform with the shared memory hierarchy (in Fig. 1(a)) and the application-specific platform with the distributed memory hierarchy (in Fig. 1(b)). There are further two variants of the shared memory hierarchy, i.e., with or without cache. On the distributed memory hierarchy, the cache is disabled. Since there are 6 kernels in both the matrix multiplication and the 2D FFT applications, 6 MicroBlaze processors are implemented on three hardware platforms as worker processors. The 6 kernels in both applications deal with independent matrices of different sizes, respectively, so that the worker processors can execute in parallel. The sizes of the local memories of the 6 MicroBlaze processors in the distributed memory hierarchy are listed in Table I. The necessary size for storing the instructions in the matrix multiplication application almost does not change for various matrix sizes because the main difference is the number of loop iterations. The local data memory needs to accommodate the two source matrices (i.e., $\mathcal{A}$ and $\mathcal{B}$ ) as well as the result matrix (i.e., $\mathcal{C}$ ). In the $2 \mathrm{D}$ FFT application, the values of $\sin \theta$ and $\cos \theta$ are determined by checking a lookup table instead of calling a math library. The size of the lookup table varies for different matrices and the lookup table itself is stored with the instructions. Therefore, the required size for the local instruction memory increases as the size of matrix grows. In the local data memory, the same 


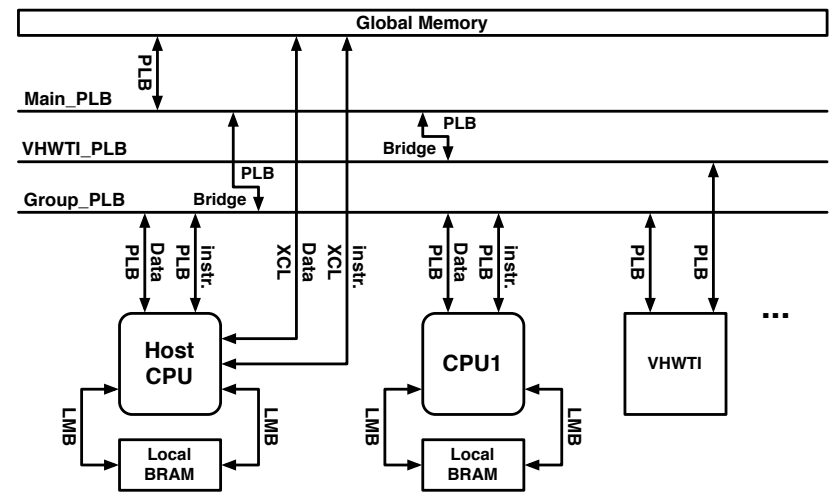

(a) Shared memory hierarchy without cache.

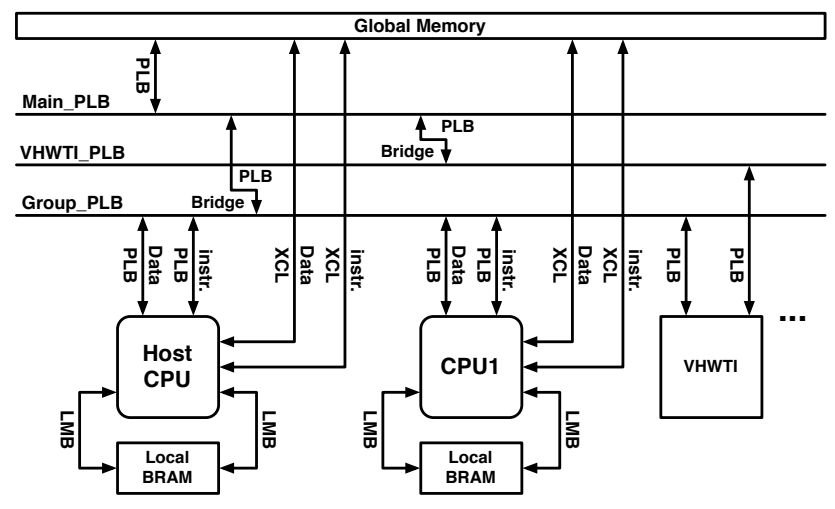

(b) Shared memory hierarchy with cache.



(c) Distributed memory hierarchy.

Figure 2. The detailed component layout and connection on Xilinx FPGA.

space is used to store both the source matrix (i.e., $\mathcal{A}$ ) and the result matrix (i.e., $\mathcal{B}$ ). On the platforms to execute the Canny edge detection algorithm, there are 4 worker processors to carry out the 4 stages of edge detection respectively. The sizes of the local instruction memory and the local data memory are shown in Table I for processing $128 \times 128$ images. These four worker processors form a processing pipeline so that 4 images can be processed simultaneously,
Table II

COMPARISON ON THE THREE PLATFORMS FOR THE THREE BENCHMARKS.

\begin{tabular}{|c|c|c|c|c|}
\hline & \multirow{2}{*}{$\begin{array}{l}\text { Memory } \\
\text { Hierarchy }\end{array}$} & \multicolumn{2}{|c|}{ Resource Requirement } & \multirow{2}{*}{$\begin{array}{l}\text { Execution } \\
\text { Time }(\mu s)\end{array}$} \\
\hline & & LUTs & BRAMs & \\
\hline \multirow{3}{*}{ MM } & Shared w/o \$ & $33,657(22.3 \%)$ & $230(55.3 \%)$ & $12,711,517$ \\
\hline & Shared $\mathrm{w} / \$^{*}$ & $39,729(26.4 \%)$ & $296(71.1 \%)$ & 323,788 \\
\hline & Distributed & $33,555(22.3 \%)$ & $100(24.0 \%)$ & 263,677 \\
\hline \multirow{3}{*}{ 2D FFT } & Shared w/o \$ & $33,657(22.3 \%)$ & $230(55.3 \%)$ & $1,431,076$ \\
\hline & Shared $\mathrm{w} /$ \$ $^{*}$ & $39,729(26.4 \%)$ & $296(71.1 \%)$ & 55,645 \\
\hline & Distributed & $39,729(26.4 \%)$ & $92(22.1 \%)$ & 35,685 \\
\hline \multirow{3}{*}{$\begin{array}{c}\text { Canny } \\
\text { Edge } \\
\text { Detection }\end{array}$} & Shared w/o \$ & $26,015(17.3 \%)$ & $169(40.6 \%)$ & $300,491,488$ \\
\hline & Shared $\mathrm{w} / \$^{*}$ & $30,810(20.4 \%)$ & $221(53.1 \%)$ & $12,575,759$ \\
\hline & Distributed & $26,633(17.7 \%)$ & $116(27.9 \%)$ & $11,136,755$ \\
\hline
\end{tabular}

${ }^{*} 1 \mathrm{~KB}$ data cache and $1 \mathrm{~KB}$ instruction cache.

Table III

COMPUTATION BREAKDOWN OF BENCHMARKS ON APPLICATION-SPECIFIC PLATFORMS (UNIT: $\mu s$ ).

\begin{tabular}{c||c|c|c|c|c}
\hline App. & $\begin{array}{c}\text { DMA-In } \\
\text { (Data) }\end{array}$ & $\begin{array}{c}\text { DMA-In } \\
\text { (Instr.) }\end{array}$ & Calculation & $\begin{array}{c}\text { DMA-Out } \\
\text { (Data) }\end{array}$ & Total \\
\hline MM & 1,082 & 232 & 261,167 & 1,196 & $\mathbf{2 6 3 , 6 7 7}$ \\
\hline 2D FFT & 774 & 354 & 33,730 & 827 & $\mathbf{3 5 , 6 8 5}$ \\
\hline
\end{tabular}

each of which is at a different stage. In the Canny edge detection application, there are 20 images to be processed in total.

The performance improvement of the application-specific platforms compared with the shared memory hierarchy without cache is remarkable, shown in Table II. The execution time is reduced by $48 \times, 40 \times$, and $27 \times$, for matrix multiplication, 2D FFT, and Canny edge detection, respectively. As expected, the performance of the shared memory hierarchy with both data and instruction caches improves significantly. However, the application-specific platform still outperforms the cache-enabled shared memory hierarchy with less resource requirement, particularly for BRAMs. The breakdown of the execution time for both matrix multiplication and 2D FFT on the application-specific platform is shown in Table III. Although the use of the distributed memory hierarchy will introduce the data/instruction transfer overhead, the overhead is very insignificant. Due to the fact that the 6 worker processors carry out the computation in parallel, the "calculation" time is the processing time of the processor that deals with the largest input data. For Canny edge detection, the 4 worker processors process 4 different images in parallel, each of which performs one stage of the algorithm. Once a worker processor finishes its work on an image, it forwards the image to the next stage in the pipeline. On the traditional shared memory platform, the image forwarding from one worker processor to another worker processor does not involve physical data transfer since all images are stored in the same shared memory. On the application-specific platform, each image forwarding includes two DMA transfers, i.e., local memory $\longrightarrow$ global memory and global memory $\longrightarrow$ local memory. 
Table V

COMPARISON BETWEEN APPLICATION-SPECIFIC PLATFORM AND TRADITIONAL PLATFORMS ON 20 PARALLEL MATRIX MULTIPLICATIONS

\begin{tabular}{|c|c|c|c|c|}
\hline \multirow{2}{*}{ Platform Type } & \multirow{2}{*}{$\begin{array}{c}\text { Number of } \\
\text { Worker Processors }\end{array}$} & \multicolumn{2}{|c|}{ Resource Requirement } & Execution \\
\hline & & LUTs & BRAMs & Time $(\mu s)$ \\
\hline Shared Memory Hierarchy w/o cache & 11 & $47,285(31.4 \%)$ & $389(93.5 \%)$ & $57,769,512$ \\
\hline Shared Memory Hierarchy w/ cache (1KB D\$ and 1KB I\$) & 6 & $39,729(26.4 \%)$ & $296(71.1 \%)$ & $1,315,016$ \\
\hline Shared Memory Hierarchy w/ cache (8KB D\$ and 8KB I\$) & 6 & $41,069(27.2 \%)$ & $314(75.5 \%)$ & $1,310,634$ \\
\hline Application-Specific (Distributed Memory Hierarchy) & 20 & $67,590(44.8 \%)$ & $404(97.1 \%)$ & $296,024^{*}$ \\
\hline
\end{tabular}

${ }^{*}$ Execution time breakdown: DMA-In(Data): 16,788 $\mu s$, DMA-In(Instructions): $740 \mu s$, Calculation: $261,084 \mu s$, DMA-Out(Data): 17,412 $\mu s$.

Table IV

PERFORMANCE COMPARISON AMONG THREE PLATFORMS FOR RUNNING CANNY EDGE DETECTION ON 20 IMAGES (UNIT: $\mu s$ ).

\begin{tabular}{|c|c|c|c|c|}
\hline \multirow{2}{*}{$\begin{array}{l}\text { Image } \\
\text { Size }\end{array}$} & \multirow{2}{*}{$\begin{array}{c}\text { Shared Memory } \\
\text { w/o Cache }\end{array}$} & \multicolumn{2}{|c|}{ Shared Memory w/ Cache } & \multirow{2}{*}{$\begin{array}{l}\text { Distributed } \\
\text { Memory }\end{array}$} \\
\hline & & 1KB Cache & $8 \mathrm{~KB}$ Cache & \\
\hline $16 \times 16$ & $4,130,124$ & 20 & 84,111 & 118,783 \\
\hline $32 \times 32$ & $24,327,878$ & 262 & 363,557 & 66 \\
\hline $64 \times 64$ & 10 & 3,7 & $1,520,418$ & $2,781,325$ \\
\hline $128 \times 128$ & $300,491,488$ & $12,575,759$ & $6,245,003$ & $11,136,755$ \\
\hline
\end{tabular}

The detailed layout of the shared memory hierarchy without cache is illustrated in Fig. 2(a). Each processor in the system is connected to a local memory for storing stack, heap, booting code, and Virtual Hardware Thread Interface (VHWTI), which is part of the hthreads system. The size of the local memory module is $64 \mathrm{~KB}$, which is typically used in our previous systems with hthreads. In the cache enabled shared memory hierarchy, shown in Fig. 2(b), both data cache and instruction cache are directly connected the global memory through the MPMC. Extra BRAM modules are needed to implement the cache and the MPMC. On the other hand, the size of the local memory in the distributed memory system shown in Fig. 2(c) is carefully selected to fit the size of corresponding data and instructions. In addition to the minimum required size of local memory listed in Table I, each local memory in the distributed memory hierarchy includes an extra $4 \mathrm{~KB}$ for storing the VHWTI. Furthermore, when using ISE to select the size of local memory, the size has to be the power of 2 . Therefore, the size of the local instruction memory for $2 \mathrm{D}$ FFT is $8 \mathrm{~KB}$ for all 6 worker processors. The size of the local data memory is $8 \mathrm{~KB}, 16 \mathrm{~KB}$, and $32 \mathrm{~KB}$ respectively. By including the required BRAM modules used by other components in the system, the application-specific platform for executing 2D FFT requires 92 BRAM modules. Similarly the applicationspecific platforms for matrix multiplication and Canny edge detection take much less BRAMs than the corresponding traditional shared memory platforms.

In the second comparison between the application-specific platform and the traditional platform based on shared memory hierarchy, the impact of the cache size on the performance is tested. Canny edge detection algorithm is applied on 20 images running on the three different platforms. 4 sets of tests are carried out with different image sizes. By observing the results in Table IV, shared memory hierarchy with large cache is even able to outperform the applicationspecific platform for Canny edge detection. The size of the local instruction memory in the distributed memory hierarchy is $4 \mathrm{~KB}$ based on the kernel size listed in Table I. An $8 \mathrm{~KB}$ cache actually is even bigger than the local instruction memory. However, MPMC has a limited number of ports, which brings the scalability issue. Large cache may unnecessarily occupy the precious BRAM resources, which may limit the number of processors an FPGA can accommodate.

In order to further demonstrate the benefit of the application-specific design flow, a parallel benchmark is designed in which 20 independent matrix multiplications are carried out. The size of all matrices is $64 \times 64$. Three different platforms are generated, as shown in Table V. In the generation of each platform, the maximum number of worker processors is used upbounded by a particular resource. On the platform using shared memory hierarchy without cache, 11 worker processors can be instantiated, which is limited by the number of available BRAMs on the Virtex-6LX240T FPGA. In order to carry out the computation of 20 matrix multiplications, two iterations have to be carried out with 11 matrix multiplications in the first iteration and 9 matrix multiplications in the second iteration. Due to the fact that both data port and instruction port of each work processor are directly connected to the PLB bus, the cache cannot be enabled. Therefore the frequent data and instruction accesses to the single shared memory result in very severe bus contention on the main PLB bus, which brings down the performance. In order to circumvent this issue, both data cache and instruction cache of each worker processor are enabled by using the Xilinx Cache Link (XCL), which has to be connected to a port of the MPMC. The current version of the MPMC has 8 ports, in which two ports are used by the main PLB bus and the host processor respectively. Therefore only 6 worker processors can be instantiated. The 20 matrix multiplications need to be carried out in 4 iterations, i.e., $6,6,6,2$. Although more iterations are needed with the cache case, the performance is improved by $44 \times$. For the matrix multiplication, the cache size has an insignificant impact on the performance. In the generation of applicationspecific platform, it is possible to instantiate 20 worker 
processors by using much smaller local memory modules than the case in traditional shared memory architecture. As a result, it only needs one iteration to complete the 20 matrix multiplications, obtaining another $4.4 \times$ speedup compared with the shared memory architecture with cache.

\section{RELATED WORK}

Before the capacity of the FPGA device became large enough to accommodate a complete MPSoC, designing hardware co-processors that were specific to respective applications is the typical approach to achieving performance improvement on FPGAs. Besides the HDLs, high-level languages, such as Impluse C [6], Handel-C [7] and MitrionC [8], are frequently used to build hardware accelerated cores. Due to the appealing feature of OpenCL in terms of architecture representation, it has been adopted in many related work to define the multicore architecture. In [9] a direct implementation of OpenCL framework on Xilinx FPGA is presented. The OpenRCL machine consists of an array or processing elements, their on-board local memory, and an off-chip global memory. This work is generalized in the following work "MARC" [10]. In MARC (Manycore Approach to Reconfigurable Computing) an application is mapped to the MARC template, which consists of one control processor and 48 algorithmic processing cores. These 48 processing cores can be parameterized to fit the application requirements. The MARC approach is similar to the approach presented in [11], in which the authors develop a tool kit for embedded designers, including compiler, mapper, designers. However, this tool is only able to statically map multiple tasks onto the many cores on an embedded SoC. In [12], the SOpenCL architectural synthesis tool is presented. The SOpenCL tool takes an OpenCL application and maps it to a custom designed hardware circuit. In this sense, it is still one variant of C-to-gate compiler, which is not the goal of this work. By contrast, the proposed design flow in this work takes one application in OpenCL and generates both the application-specific system platform and the corresponding software executable. We focus on generating the application-specific multi/many-core platform by mapping the application kernels to processing cores and designing optimal memory hierarchy and interconnect.

\section{CONCLUSIONS}

In this work a design flow for developing applicationspecific platform on million-LUT FPGAs is presented. Given an application written in OpenCL, a hardware platform consisting of one host processor and multiple worker processors is generated. Each OpenCL kernel is mapped to one separate worker processor to maximize the processing parallelism. A distributed memory hierarchy is applied in which each worker processor is equipped with separate local instruction memory and local data memory. The size of each local memory is determined by analyzing the corresponding OpenCL kernel so that it is application-specific and resource efficient. Significant performance improvements have been achieved for multiple benchmarks running on the application-specific platforms. Future work will keep improving the design flow for generating optimal application-specific platforms by considering additional components, such as memory hierarchy, the interconnect, and the types of worker processors, etc.

\section{REFERENCES}

[1] S. Ma, M. Huang, E. Cartwright, and D. Andrews, "Scalable memory hierarchies for embedded manycore systems," in Proc. 8th International Symposium on Applied Reconfigurable Computing (ARC 2012), LNCS 7199, Mar. 2012, pp. 151-162.

[2] Open Computing Language (OpenCL). http://www.khronos.org/opencl.

[3] W. Peck, E. Anderson, J. Agron, J. Stevens, F. Baijot, and D. Andrews, "Hthreads: A computational model for reconfigurable devices," in Proc. 16th International Conference on Field Programmable Logic and Applications (FPL'06), Aug. 2006, pp. 885-888.

[4] E. Cartwright, S. Ma, D. Andrews, and M. Huang, "Creating HW/SW co-designed MPSoPC's from high level programming models," in Proc. Workshop on Multiprocessor Systems on (Programmable) Chips (MPSoC 2011), July 2011, pp. $554-560$.

[5] J. Canny, "A computational approach to edge detection," IEEE Trans. Pattern Anal. Machine Intell., vol. 8, no. 6, pp. 679698, Nov. 1986.

[6] Impulse C-http://www.impulseaccelerated.com, Impulse Accelerated Technologies, Inc., last accessed October 5, 2012.

[7] Handel-C - http://www.mentor.com/products/fpga/handel-c, Mentor Graphics Corp., last accessed October 5, 2012.

[8] Mitrion-C - http://www.mitrionics.com, Mitrionics AB, last accessed October 5, 2012.

[9] M. Lin, I. Lebedev, and J. Wawrzynek, "OpenRCL: Lowpower high-performance computing with reconfigurable devices," in Proc. 20th International Conference on Field Programmable Logic and Applications (FPL 2010), Aug. 2010, pp. 458-463.

[10] I. Lebedev, S. Cheng, A. Doupnik, J. Martin, C. Fletcher, D. Burke, M. Lin, and J. Wawrzynek, "MARC: A manycore approach to reconfigurable computing," in Proc. 2010 International Conference on Reconfigurable Computing and FPGAs (ReConFig'10), Dec. 2010, pp. 7-12.

[11] H. Tomiyama, "Challenges of programming embedded manycore SoCs with OpenCL," in Proc. 11th International Forum on Embedded MPSoC and Multicore (MPSoC'11), July 2011.

[12] M. Owaida, N. Bellas, K. Daloukas, and C. D. Antonopoulos, "Synthesis of platform architectures from OpenCL programs," in Proc. 19th Annual International Symposium on FieldProgrammable Custom Computing Machines (FCCM'11), May 2011, pp. 186-193. 